$\mathrm{J}$ o u r n a l of

Mathematics

and Applications

JMA No 42, pp 135-146 (2019)

\title{
Number of Zeros of a Polynomial in a Specific Region with Restricted Coefficients
}

\author{
Abdullah Mir, Abrar Ahmad and Adil Hussain Malik
}

ABstract: This paper focuses on the problem concerning the location and the number of zeros of polynomials in a specific region when their coefficients are restricted with special conditions. We obtain extensions of some classical results concerning the number of zeros of polynomials in a prescribed region by imposing the restrictions on the moduli of the coefficients, the real parts(only) of the coefficients, and the real and imaginary parts of the coefficients.

AMS Subject Classification: 30A99, 30E10, 41A10.

Keywords and Phrases: Polynomial; Zeros; Eneström-Kakeya theorem.

\section{Introduction}

Locating zeros of polynomials with special conditions for the coefficients, in particular, the number of zeros of complex polynomials in a disk when their coefficients are restricted with special conditions has applications in many areas of applied mathematics, including linear control systems, electrical networks, root approximation and signal processing, and for this reason there is always a need for better and better estimates for the region containing some or all the zeros of a polynomial. A review on the location of zeros of polynomials can be found in ([1], [5], [8], [11]). If $P(z)=\sum_{j=0}^{n} a_{j} z^{j}$ is a polynomial of degree $n$ such that $a_{n} \geq a_{n-1} \geq \ldots \geq a_{1} \geq a_{0}>0$, then $P(z)$ has all its zeros in $|z| \leq 1$. This famous result is known as Eneström-Kakeya theorem, for reference see (section 8.3 of [11]). In the literature, for example see ([1] - [12]), there exist various extensions and generalizations of Eneström-Kakeya theorem. Taking

COPYRIGHT (c) by Publishing House of Rzeszów University of Technology P.O. Box 85, 35-959 Rzeszów, Poland 
account of the restrictions on the coefficients of a polynomial allows for establishing improved bounds and here, in this paper, we impose some restrictions on the coefficients of polynomials in order to count the number of zeros in a specific region. The following result concerning the number of zeros of a polynomial in a closed disk can be found in Titchmarsh's classic "The Theory of Functions", see ([13], page 171, 2nd edition).

Theorem A. Let $F(z)$ be analytic in $|z| \leq R$. Let $|F(z)| \leq M$ in $|z| \leq R$ and suppose $F(0) \neq 0$. Then for $0<\delta<1$, the number of zeros of $F(z)$ in the disk $|z| \leq R \delta$ does not exceed

$$
\frac{1}{\log \frac{1}{\delta}} \log \frac{M}{|F(0)|} .
$$

Regarding the number of zeros in $|z| \leq \frac{1}{2}$ and by putting a restriction on the coefficients of a polynomial similar to that of the Eneström-Kakeya theorem, Mohammad [9] used a special case of Theorem A to prove the following result.

Theorem B. If $P(z)=\sum_{j=0}^{n} a_{j} z^{j}$ is a polynomial of degree $n$ such that $0<a_{0} \leq$ $a_{1} \leq \ldots \leq a_{n}$, then the number of zeros of $P(z)$ in $|z| \leq \frac{1}{2}$ does not exceed

$$
1+\frac{1}{\log 2} \log \left(\frac{a_{n}}{a_{0}}\right)
$$

The above result of Mohammad [9] was generalized in different ways for example see ([1], [2], [4], [5], [11]). Using hypotheses related to those of Theorem B, very recently Qasim et al. [6] imposed a monotonic condition on the moduli and then on the real and imaginary parts of the coefficients of the Lucanary type of polynomials $P(z)=a_{0}+\sum_{j=\mu}^{n} a_{j} z^{j}$ and proved the following results.

Theorem C. Let $P(z)=a_{0}+\sum_{j=\mu}^{n} a_{j} z^{j}, 1 \leq \mu \leq n-1, a_{0} \neq 0$ be a polynomial of degree $n$. If for some real $\alpha$ and $\beta$

$$
\left|\arg a_{j}-\beta\right| \leq \alpha \leq \frac{\pi}{2}, \quad \mu \leq j \leq n,
$$

and for some $t>0$ and some $k$ with $\mu \leq k \leq n$,

$$
t^{\mu}\left|a_{\mu}\right| \leq \ldots \leq t^{k-1}\left|a_{k-1}\right| \leq t^{k}\left|a_{k}\right| \geq t^{k+1}\left|a_{k+1}\right| \geq \ldots \geq t^{n-1}\left|a_{n-1}\right| \geq t^{n}\left|a_{n}\right|,
$$

then the number of zeros of $P(z)$ in $|z| \leq \frac{1}{2}$ does not exceed

$$
\frac{1}{\log 2} \log \left(\frac{M}{\left|a_{0}\right|}\right)
$$

where

$$
\begin{gathered}
M=2\left|a_{0}\right| t+\left|a_{\mu}\right| t^{\mu+1}(1-\sin \alpha-\cos \alpha)+2\left|a_{k}\right| t^{k+1} \cos \alpha+ \\
\left|a_{n}\right| t^{n+1}(1-\sin \alpha-\cos \alpha)+2 \sum_{j=\mu}^{n}\left|a_{j}\right| t^{j+1} \sin \alpha .
\end{gathered}
$$


Theorem D. Let $P(z)=a_{0}+\sum_{j=\mu}^{n} a_{j} z^{j}, 1 \leq \mu \leq n-1, a_{0} \neq 0$ be a polynomial of degree $n$ with Re $a_{j}=\alpha_{j}$ and $\operatorname{Im} a_{j}=\beta_{j}$ for $\mu \leq j \leq n$. Suppose that for some $t>0$ and some $k$ with $\mu \leq k \leq n$, we have

$$
t^{\mu} \alpha_{\mu} \leq \ldots \leq t^{k-1} \alpha_{k-1} \leq t^{k} \alpha_{k} \geq t^{k+1} \alpha_{k+1} \geq \ldots \geq t^{n-1} \alpha_{n-1} \geq t^{n} \alpha_{n},
$$

then the number of zeros of $P(z)$ in $|z| \leq \frac{t}{2}$ does not exceed

$$
\frac{1}{\log 2} \log \left(\frac{M}{\left|a_{0}\right|}\right)
$$

where

$$
\begin{aligned}
M= & 2\left(\left|\alpha_{0}\right|+\left|\beta_{0}\right|\right) t+\left(\left|a_{\mu}\right|-\alpha_{\mu}\right) t^{\mu+1}+2\left|\alpha_{k}\right| t^{k+1}+ \\
& \left(\left|\alpha_{n}\right|-\alpha_{n}\right) t^{n+1}+2 \sum_{j=\mu}^{n}\left|\beta_{j}\right| t^{j+1} .
\end{aligned}
$$

Theorem E. Let $P(z)=a_{0}+\sum_{j=\mu}^{n} a_{j} z^{j}, 1 \leq \mu \leq n-1, a_{0} \neq 0$ be a polynomial of degree $n$ with Re $a_{j}=\alpha_{j}$ and Im $a_{j}=\beta_{j}$ for $\mu \leq j \leq n$. Suppose that for some $t>0$ and some $k$ with $\mu \leq k \leq n$, we have

$$
t^{\mu} \alpha_{\mu} \leq \ldots \leq t^{k-1} \alpha_{k-1} \leq t^{k} \alpha_{k} \geq t^{k+1} \alpha_{k+1} \geq \ldots \geq t^{n-1} \alpha_{n-1} \geq t^{n} \alpha_{n}
$$

and for some $\mu \leq l \leq n$ we have

$$
t^{\mu} \beta_{\mu} \leq \ldots \leq t^{l-1} \beta_{l-1} \leq t^{k} \beta_{l} \geq t^{l+1} \beta_{l+1} \geq \ldots \geq t^{n-1} \beta_{n-1} \geq t^{n} \beta_{n},
$$

then the number of zeros of $P(z)$ in $|z| \leq \frac{t}{2}$ does not exceed

$$
\frac{1}{\log 2} \log \left(\frac{M}{\left|a_{0}\right|}\right)
$$

where

$$
\begin{aligned}
M= & 2\left(\left|\alpha_{0}\right|+\left|\beta_{0}\right|\right) t+\left(\left|a_{\mu}\right|-\alpha_{\mu}+\left|\beta_{\mu}\right|-\beta_{\mu}\right) t^{\mu+1} \\
& +2\left(\alpha_{k} t^{k+1}+\beta_{l} t^{l+1}\right)+\left(\left|\alpha_{n}\right|-\alpha_{n}+\left|\beta_{\mu}\right|-\beta_{\mu}\right) t^{n+1} .
\end{aligned}
$$

In this paper, we further weaken the hypotheses of the above results and prove the following. 


\section{Main results}

Theorem 1. Let $P(z)=a_{0}+\sum_{j=\mu}^{n} a_{j} z^{j}, 1 \leq \mu<n, a_{0} \neq 0$, where for some $t>0$ and some $\mu \leq k \leq n$,

$$
t^{\mu}\left|a_{\mu}\right| \leq \ldots \leq t^{k-1}\left|a_{k-1}\right| \leq t^{k}\left|a_{k}\right| \geq t^{k+1}\left|a_{k+1}\right| \geq \ldots \geq t^{n-1}\left|a_{n-1}\right| \geq t^{n}\left|a_{n}\right|
$$

and $\left|\arg a_{j}-\beta\right| \leq \alpha \leq \frac{\pi}{2}$ for $\mu \leq j \leq n$, for some real $\alpha$ and $\beta$. Then for $0<\delta<1$, the number of zeros of $P(z)$ in the disk $|z| \leq \delta t$ does not exceed

$$
\frac{1}{\log \frac{1}{\delta}} \log \frac{M}{\left|a_{0}\right|},
$$

where

$$
\begin{aligned}
M= & 2\left|a_{0}\right| t+\left(\left|a_{\mu}\right| t^{\mu+1}+\left|a_{n}\right| t^{n+1}\right)(1-\cos \alpha-\sin \alpha) \\
& +2\left|a_{k}\right| t^{k+1} \cos \alpha+2 \sum_{j=\mu}^{n}\left|a_{j}\right| t^{j+1} \sin \alpha .
\end{aligned}
$$

Notice that when $t=1$ in Theorem 1 , we get the following.

Corollary 1. Let $P(z)=a_{0}+\sum_{j=\mu}^{n} a_{j} z^{j}, 1 \leq \mu<n, a_{0} \neq 0$, where for some $\mu \leq k \leq n$,

$$
\left|a_{\mu}\right| \leq \ldots \leq\left|a_{k-1}\right| \leq\left|a_{k}\right| \geq\left|a_{k+1}\right| \geq \ldots \geq\left|a_{n-1}\right| \geq\left|a_{n}\right|
$$

and $\left|\arg a_{j}-\beta\right| \leq \alpha \leq \frac{\pi}{2}$ for $\mu \leq j \leq n$, for some real $\alpha$ and $\beta$. Then for $0<\delta<1$, the number of zeros of $P(z)$ in the disk $|z| \leq \delta$ does not exceed

$$
\frac{1}{\log \frac{1}{\delta}} \log \frac{M}{\left|a_{0}\right|},
$$

where

$$
\begin{aligned}
M= & 2\left|a_{0}\right|+\left(\left|a_{\mu}\right|+\left|a_{n}\right|\right)(1-\cos \alpha-\sin \alpha) \\
& +2\left|a_{k}\right| \cos \alpha+2 \sum_{j=\mu}^{n}\left|a_{j}\right| \sin \alpha .
\end{aligned}
$$

Clearly for $\delta=\frac{1}{2}$, Theorem 1 reduces to Theorem C and Corollary 1 reduces to Corollary 1.1 of Qasim et al. [6]. With $t=1$ and $k=n$ in Theorem 1, the hypothesis becomes $\left|a_{\mu}\right| \leq \ldots \leq\left|a_{n-1}\right| \leq\left|a_{n}\right|$, and the value of M becomes $2\left|a_{0}\right|+\left(\left|a_{\mu}\right|+\left|a_{n}\right|\right)(1-$ $\cos \alpha-\sin \alpha)+2\left|a_{n}\right| \cos \alpha+2 \sum_{j=\mu}^{n}\left|a_{j}\right| \sin \alpha$, and hence Theorem 1 implies Corollary 1.2 of Qasim et al. [6]. In the same way for $t=1, k=\mu$ and for $\delta=\frac{1}{2}$, Theorem 1 implies Corollary 1.3 of Qasim et al. [6]. 
Number of Zeros of a Polynomial ina Specific Region with Restricted Coefficients139

Theorem 2. Let $P(z)=a_{0}+\sum_{j=\mu}^{n} a_{j} z^{j}, 1 \leq \mu<n, a_{0} \neq 0$, where Re $a_{j}=\alpha_{j}$ and Im $a_{j}=\beta_{j}$ for $\mu \leq j \leq n$. Suppose that for some $t>0$ and some $k$ with $\mu \leq k \leq n$, we have

$$
t^{\mu} \alpha_{\mu} \leq \ldots \leq t^{k-1} \alpha_{k-1} \leq t^{k} \alpha_{k} \geq t^{k+1} \alpha_{k+1} \geq \ldots \geq t^{n-1} \alpha_{n-1} \geq t^{n} \alpha_{n}
$$

Then for $0<\delta<1$, the number of zeros of $P(z)$ in the disk $|z| \leq \delta t$ does not exceed

$$
\frac{1}{\log \frac{1}{\delta}} \log \frac{M}{\left|a_{0}\right|}
$$

where

$$
\begin{aligned}
M= & 2\left(\left|\alpha_{0}\right|+\left|\beta_{0}\right|\right) t+\left(\left|\alpha_{\mu}\right|-\alpha_{\mu}\right) t^{\mu+1} \\
& +2 \alpha_{k} t^{k+1}+\left(\left|\alpha_{n}\right|-\alpha_{n}\right) t^{n+1}+2 \sum_{j=\mu}^{n}\left|\beta_{j}\right| t^{j+1} .
\end{aligned}
$$

Remark 1. For $\delta=\frac{1}{2}$, Theorem 2 reduces to Theorem D.

Notice that with $t=1$ in Theorem 2 , we get the following.

Corollary 2. Let $P(z)=a_{0}+\sum_{j=\mu}^{n} a_{j} z^{j}, 1 \leq \mu<n, a_{0} \neq 0$, where Re $a_{j}=\alpha_{j}$ and Im $a_{j}=\beta_{j}$ for $\mu \leq j \leq n$. Suppose we have for some $\mu \leq k \leq n$,

$$
\alpha_{\mu} \leq \ldots \leq \alpha_{k-1} \leq \alpha_{k} \geq \alpha_{k+1} \geq \ldots \geq \alpha_{n-1} \geq \alpha_{n}
$$

Then for $0<\delta<1$, the number of zeros of $P(z)$ in the disk $|z|<\delta$ does not exceed

$$
\frac{1}{\log \frac{1}{\delta}} \log \frac{M}{\left|a_{0}\right|}
$$

where $M=2\left(\left|\alpha_{0}\right|+\left|\beta_{0}\right|\right)+\left(\left|\alpha_{\mu}\right|-\alpha_{\mu}\right)+2 \alpha_{k}+\left(\left|\alpha_{n}\right|-\alpha_{n}\right)+2 \sum_{j=\mu}^{n}\left|\beta_{j}\right|$.

Clearly for $\delta=\frac{1}{2}$, the Corollary 2 reduces to Corollary 2.1 of Qasim et al. [6].

With $t=1, k=n$ in Theorem 2, the hypothesis becomes $\alpha_{\mu} \leq \ldots \leq \alpha_{n-1} \leq \alpha_{n}, 1 \leq$ $\mu<n$ and the value of $M$ becomes

$$
2\left(\left|\alpha_{0}\right|+\left|\beta_{0}\right|\right)+\left(\left|\alpha_{\mu}\right|-\alpha_{\mu}\right)+\left(\left|\alpha_{n}\right|+\alpha_{n}\right)+2 \sum_{j=\mu}^{n}\left|\beta_{j}\right|,
$$

therefore, Corollary 2.2 of Qasim et al. [6] follows from Theorem 2.

By manipulating the parameter $k, \mu$ and $t$, we easily get Corollary 2.3 and Corollary 2.4 of Qasim et al. [6] from Theorem 2.

Finally, we put the monotonicity-type condition on the real and imaginary parts of 
the coefficient of $P(z)=a_{0}+\sum_{j=\mu}^{n} a_{j} z^{j}$ and get the following result.

Theorem 3. Let $P(z)=a_{0}+\sum_{j=\mu}^{n} a_{j} z^{j}, 1 \leq \mu<n, a_{0} \neq 0$ where Re $a_{j}=\alpha_{j}$ and Im $a_{j}=\beta_{j}$ for $\mu \leq j \leq n$. Suppose that for some $t>0$, for some $\mu \leq k \leq n$, we have

$$
t^{\mu} \alpha_{\mu} \leq \ldots \leq t^{k-1} \alpha_{k-1} \leq t^{k} \alpha_{k} \geq t^{k+1} \alpha_{k+1} \geq \ldots \geq t^{n-1} \alpha_{n-1} \geq t^{n} \alpha_{n}
$$

and for some $\mu \leq l \leq n$, we have

$$
t^{\mu} \beta_{\mu} \leq \ldots \leq t^{l-1} \beta_{l-1} \leq t^{k} \beta_{l} \geq t^{l+1} \beta_{l+1} \geq \ldots \geq t^{n-1} \beta_{n-1} \geq t^{n} \beta_{n} .
$$

Then for $0<\delta<1$, the number of zeros of $P(z)$ in the disk $|z| \leq \delta t$ does not exceed

$$
\frac{1}{\log \frac{1}{\delta}} \log \frac{M}{\left|a_{0}\right|},
$$

where

$$
\begin{aligned}
M= & 2\left(\left|\alpha_{0}\right|+\left|\beta_{0}\right|\right) t+\left(\left|a_{\mu}\right|-\alpha_{\mu}+\left|\beta_{\mu}\right|-\beta_{\mu}\right) t^{\mu+1} \\
& +2\left(\alpha_{k} t^{k+1}+\beta_{l} t^{l+1}\right)+\left(\left|\alpha_{n}\right|-\alpha_{n}+\left|\beta_{\mu}\right|-\beta_{\mu}\right) t^{n+1} .
\end{aligned}
$$

Taking $\delta=\frac{1}{2}$ in Theorem 3, we get Theorem E. Theorem 3 gives several corollaries with hypotheses concerning monotonicity of real and imaginary parts. For example, with $t=1$, we have the following result.

Corollary 3. Let $P(z)=a_{0}+\sum_{j=\mu}^{n} a_{j} z^{j}, 1 \leq \mu<n, a_{0} \neq 0$, where Re $a_{j}=\alpha_{j}$ and Im $a_{j}=\beta_{j}$ for $\mu \leq j \leq n$. Suppose that for some $\mu \leq k \leq n$, we have

$$
\alpha_{\mu} \leq \ldots \leq \alpha_{k-1} \leq \alpha_{k} \geq \alpha_{k+1} \geq \ldots \geq \alpha_{n-1} \geq \alpha_{n}
$$

and for some $\mu \leq l \leq n$, we have

$$
\beta_{\mu} \leq \ldots \leq \beta_{l-1} \leq \beta_{l} \geq \beta_{l+1} \geq \ldots \geq \beta_{n-1} \geq \beta_{n} .
$$

Then for $0<\delta<1$, the number of zeros of $P(z)$ in the disk $|z| \leq \delta$ does not exceed

$$
\frac{1}{\log \frac{1}{\delta}} \log \frac{M}{\left|a_{0}\right|},
$$

where

$M=2\left(\left|\alpha_{0}\right|+\left|\beta_{0}\right|\right)+\left(\left|a_{\mu}\right|-\alpha_{\mu}+\left|\beta_{\mu}\right|-\beta_{\mu}\right)+2\left(\alpha_{k}+\beta_{l}\right)+\left(\left|\alpha_{n}\right|-\alpha_{n}+\left|\beta_{\mu}\right|-\beta_{\mu}\right)$.

With $t=1$ and $k=l=n$ in Theorem 3, we get the following. 
Number of Zeros of a Polynomial ina Specific Region with Restricted Coefficients141

Corollary 4. Let $P(z)=a_{0}+\sum_{j=\mu}^{n} a_{j} z^{j}, 1 \leq \mu<n, a_{0} \neq 0$, where Re $a_{j}=\alpha_{j}$ and Im $a_{j}=\beta_{j}$ for $\mu \leq j \leq n$. Suppose that for some $\mu \leq k \leq n$, we have

$$
\alpha_{\mu} \leq \ldots \leq \alpha_{n-1} \leq \alpha_{n}
$$

and

$$
\beta_{\mu} \leq \ldots \leq \beta_{n-1} \leq \beta_{n}
$$

Then for $0<\delta<1$, the number of zeros of $P(z)$ in the disk $|z| \leq \delta$ does not exceed

$$
\frac{1}{\log \frac{1}{\delta}} \log \frac{M}{\left|a_{0}\right|},
$$

where

$$
M=2\left(\left|\alpha_{0}\right|+\left|\beta_{0}\right|\right)+\left(\left|a_{\mu}\right|-\alpha_{\mu}+\left|\beta_{\mu}\right|-\beta_{\mu}\right)+\left(\left|\alpha_{n}\right|+\alpha_{n}+\left|\beta_{\mu}\right|+\beta_{\mu}\right) .
$$

For $t=1, k=l=\mu$ in Theorem 3 , we get the following

Corollary 5. Let $P(z)=a_{0}+\sum_{j=\mu}^{n} a_{j} z^{j}, 1 \leq \mu<n, a_{0} \neq 0$, where Re $a_{j}=\alpha_{j}$ and $\operatorname{Im} a_{j}=\beta_{j}$ for $\mu \leq j \leq n$. Suppose that

$$
\alpha_{\mu} \geq \ldots \geq \alpha_{n-1} \geq \alpha_{n}
$$

and

$$
\beta_{\mu} \geq \ldots \geq \beta_{n-1} \geq \beta_{n}
$$

Then for $0<\delta<1$, the number of zeros of $P(z)$ in the disk $|z| \leq \delta$ does not exceed

$$
\frac{1}{\log \frac{1}{\delta}} \log \frac{M}{\left|a_{0}\right|},
$$

where

$$
M=2\left(\left|\alpha_{0}\right|+\left|\beta_{0}\right|\right)+\left(\left|a_{\mu}\right|+\alpha_{\mu}+\left|\beta_{\mu}\right|+\beta_{\mu}\right)+\left(\left|\alpha_{n}\right|-\alpha_{n}+\left|\beta_{\mu}\right|-\beta_{\mu}\right) .
$$

\section{Proofs of theorems}

We need the following lemma for the proofs of theorems.

Lemma 1. For any two complex numbers $b_{0}$ and $b_{1}$ such that $\left|b_{0}\right| \geq\left|b_{1}\right|$. Suppose $\left|\arg b_{j}-\beta\right| \leq \alpha \leq \frac{\pi}{2}$, for $j=0,1$ for some real $\alpha$ and $\beta$, then

$$
\left|b_{0}-b_{1}\right| \leq\left(\left|b_{0}\right|-\left|b_{1}\right|\right) \cos \alpha+\left(\left|b_{0}\right|+\left|b_{1}\right|\right) \sin \alpha \text {. }
$$


The above lemma is due to Govil and Rahman [5].

Proof of Theorem 1. Consider the polynomial

$$
\begin{aligned}
F(z) & =(t-z) P(z) \\
& =(t-z)\left(a_{0}+\sum_{j=\mu}^{n} a_{j} z^{j}\right) \\
& =a_{0} t+\sum_{j=\mu}^{n} t a_{j} z^{j}-a_{0} z-\sum_{j=\mu}^{n} a_{j} z^{j+1} \\
& =a_{0}(t-z)+\sum_{j=\mu}^{n} t a_{j} z^{j}-\sum_{j=\mu+1}^{n+1} a_{j-1} z^{j} \\
& =a_{0}(t-z)+t a_{\mu} z^{\mu}+\sum_{j=\mu+1}^{n}\left(t a_{j}-a_{j-1}\right) z^{j}-a_{n} z^{n+1} .
\end{aligned}
$$

For $|z|=t$, we have

$$
\begin{aligned}
|F(z)| & \leq 2 t\left|a_{0}\right|+\left|a_{\mu}\right| t^{\mu+1}+\sum_{j=\mu+1}^{n}\left|t a_{j}-a_{j-1}\right| t^{j}+\left|a_{n}\right| t^{n+1} \\
& =2 t\left|a_{0}\right|+\left|a_{\mu}\right| t^{\mu+1}+\sum_{j=\mu+1}^{k}\left|t a_{j}-a_{j-1}\right| t^{j}+\sum_{j=k+1}^{n}\left|a_{j-1}-t a_{j}\right| t^{j}+\left|a_{n}\right| t^{n+1} .
\end{aligned}
$$

Using Lemma 1 with $b_{0}=a_{j} t$ and $b_{1}=a_{j-1}$ when $\mu+1 \leq j \leq k$ and with $b_{0}=a_{j-1}$ and $b_{1}=a_{j} t$ when $k+1 \leq j \leq n$,

$$
\begin{aligned}
|F(z)| \leq & 2 t\left|a_{0}\right|+\left|a_{\mu}\right| t^{\mu+1}+\sum_{j=\mu+1}^{k}\left\{\left(\left|a_{j}\right| t-\left|a_{j-1}\right|\right) \cos \alpha+\left(\left|a_{j}\right| t+\left|a_{j-1}\right|\right) \sin \alpha\right\} t^{j} \\
& +\sum_{j=k+1}^{n}\left\{\left(\left|a_{j-1}\right|-\left|a_{j}\right| t\right) \cos \alpha+\left(\left|a_{j}\right| t+\left|a_{j-1}\right|\right) \sin \alpha\right\} t^{j}+\left|a_{n}\right| t^{n+1} \\
& =2\left|a_{0}\right| t+\left|a_{\mu}\right| t^{\mu+1}+\sum_{j=\mu+1}^{k}\left|a_{j}\right| t^{j+1} \cos \alpha-\sum_{j=\mu+1}^{k}\left|a_{j-1}\right| t^{j} \cos \alpha \\
& +\sum_{j=\mu+1}^{k}\left|a_{j}\right| t^{j+1} \sin \alpha+\sum_{j=\mu+1}^{k}\left|a_{j-1}\right| t^{j} \sin \alpha+\sum_{j=k+1}^{n}\left|a_{j-1}\right| t^{j} \cos \alpha \\
& -\sum_{j=k+1}^{n}\left|a_{j}\right| t^{j+1} \cos \alpha+\sum_{j=k+1}^{n}\left|a_{j-1}\right| t^{j} \sin \alpha+\sum_{j=k+1}^{n}\left|a_{j}\right| t^{j+1} \sin \alpha+\left|a_{n}\right| t^{n+1}
\end{aligned}
$$


Number of Zeros of a Polynomial ina Specific Region with Restricted Coefficients143

$$
\begin{aligned}
& =2\left|a_{0}\right| t+\left|a_{\mu}\right| t^{\mu+1}-\left|a_{\mu}\right| t^{\mu+1} \cos \alpha+\left|a_{k}\right| t^{k+1} \cos \alpha+\left|a_{\mu}\right| t^{\mu+1} \sin \alpha \\
& +\left|a_{k}\right| t^{k+1} \sin \alpha+2 \sum_{j=\mu+1}^{k-1}\left|a_{j}\right| t^{j+1} \sin \alpha+\left|a_{k}\right| t^{k+1} \cos \alpha-\left|a_{n}\right| t^{n+1} \cos \alpha+\left|a_{k}\right| t^{k+1} \sin \alpha \\
& +\left|a_{n}\right| t^{n+1} \sin \alpha+2 \sum_{j=k+1}^{n-1}\left|a_{j}\right| t^{j+1} \sin \alpha+\left|a_{n}\right| t^{n+1} \\
& =2\left|a_{0}\right| t+\left|a_{\mu}\right| t^{\mu+1}+\left|a_{\mu}\right| t^{\mu+1}(\sin \alpha-\cos \alpha)+2 \sum_{j=\mu+1}^{n-1}\left|a_{j}\right| t^{j+1} \sin \alpha \\
& +2\left|a_{k}\right| t^{k+1} \cos \alpha+(\sin \alpha-\cos \alpha+1)\left|a_{n}\right| t^{n+1} \\
& =2\left|a_{0}\right| t+\left|a_{\mu}\right| t^{\mu+1}(1-\sin \alpha-\cos \alpha)+2\left|a_{k}\right| t^{k+1} \cos \alpha+\left|a_{n}\right| t^{n+1}(1-\sin \alpha-\cos \alpha) \\
& +2 \sum_{j=\mu}^{n}\left|a_{j}\right| t^{j+1} \sin \alpha . \\
& =M(\text { say }) .
\end{aligned}
$$

Now $F(z)$ is analytic in $|z| \leq t$ and $|F(z)| \leq M$ for $|z|=t$. So by Theorem A and the Maximum Modulus Theorem, the number of zeros of $F$ (and hence of $P$ ) in $|z| \leq \delta t$ is less than or equal to

$$
\frac{1}{\log \frac{1}{\delta}} \log \left(\frac{M}{\left|a_{0}\right|}\right) .
$$

Hence the Theorem 1 follows.

Proof of Theorem 2. As in the proof of Theorem 1,

$$
\begin{aligned}
F(z) & =(t-z) P(z) \\
& =a_{0}(t-z)+t a_{\mu} z^{\mu}+\sum_{j=\mu+1}^{n}\left(t a_{j}-a_{j-1}\right) z^{j}-a_{n} z^{n+1},
\end{aligned}
$$

and so

$$
\begin{aligned}
F(z) & =\left(\alpha_{0}+i \beta_{0}\right)(t-z)+\left(\alpha_{\mu}+i \beta_{\mu}\right) t z^{\mu}+\sum_{j=\mu+1}^{n}\left(\alpha_{j} t-\alpha_{j-1}\right) z^{j} \\
& +i \sum_{j=\mu+1}^{n}\left(\beta_{j} t-\beta_{j-1}\right) z^{j}-\left(\alpha_{n}+i \beta_{n}\right) z^{n+1} .
\end{aligned}
$$


For $|z|=t$, we have

$$
\begin{aligned}
|F(z)| \leq & 2\left(\left|\alpha_{0}\right|+\left|\beta_{0}\right|\right) t+\left(\left|\alpha_{\mu}\right|+\left|\beta_{\mu}\right|\right) t^{\mu+1}+\sum_{j=\mu+1}^{n}\left|\alpha_{j} t-\alpha_{j-1}\right| t^{j} \\
& +\sum_{j=\mu+1}^{n}\left(\left|\beta_{j}\right| t+\left|\beta_{j-1}\right|\right) t^{j}+\left(\left|\alpha_{n}\right|+\left|\beta_{n}\right|\right) t^{n+1} \\
& =2\left(\left|\alpha_{0}\right|+\left|\beta_{0}\right|\right) t+\left(\left|\alpha_{\mu}\right|+\left|\beta_{\mu}\right|\right) t^{\mu+1}+\sum_{j=\mu+1}^{n}\left(\alpha_{j} t-\alpha_{j-1}\right) t^{j} \\
& +\sum_{j=k+1}^{n}\left(\alpha_{j-1}-\alpha_{j} t\right) t^{j}+\left|\beta_{\mu}\right| t^{\mu+1}+2 \sum_{j=\mu+1}^{n-1}\left|\beta_{j}\right| t^{j+1}+\left|\beta_{n}\right| t^{n+1} \\
& +\left(\left|\alpha_{n}\right|+\left|\beta_{n}\right|\right) t^{n+1} \\
& =2\left(\left|\alpha_{0}\right|+\left|\beta_{0}\right|\right) t+\left(\left|\alpha_{\mu}\right|-\alpha_{\mu}\right) t^{\mu+1}+2 \alpha_{k} t^{k+1}+\left(\left|\alpha_{n}\right|-\alpha_{n}\right) t^{n+1} \\
& +2 \sum_{j=\mu}^{n}\left|\beta_{j}\right| t^{j+1} \\
& =M
\end{aligned}
$$

The result follows as in the proof of Theorem 1.

Proof of Theorem 3. As in the proof of Theorem 2,

$$
\begin{aligned}
F(z) & =(t-z) P(z) \\
& =a_{0}(t-z)+t a_{\mu} z^{\mu}+\sum_{j=\mu+1}^{n}\left(a_{j} t-a_{j-1}\right) z^{j}-a_{n} z^{n+1} \\
& =\left(\alpha_{0}+i \beta_{0}\right)(t-z)+\left(\alpha_{\mu}+i \beta_{\mu}\right) t z^{\mu}+\sum_{j=\mu+1}^{n}\left(\alpha_{j} t-\alpha_{j-1}\right) z^{j} \\
& +i \sum_{j=\mu+1}^{n}\left(\beta_{j} t-\beta_{j-1}\right) z^{j}-\left(\alpha_{n}+i \beta_{n}\right) z^{n+1} .
\end{aligned}
$$

For $|z|=t$, we have

$$
\begin{aligned}
|F(z)| & \leq 2\left(\left|\alpha_{0}\right|+\left|\beta_{0}\right|\right) t+\left(\left|\alpha_{\mu}\right|+\left|\beta_{\mu}\right|\right) t^{\mu+1}+\sum_{j=\mu+1}^{n}\left|\alpha_{j} t-\alpha_{j-1}\right| t^{j} \\
& +\sum_{j=\mu+1}^{n}\left|\beta_{j} t-\beta_{j-1}\right| t^{j}+\left(\left|\alpha_{n}\right|+\left|\beta_{n}\right|\right) t^{n+1}
\end{aligned}
$$


Number of Zeros of a Polynomial ina Specific Region with Restricted Coefficients145

$$
\begin{aligned}
& =2\left(\left|\alpha_{0}\right|+\left|\beta_{0}\right|\right) t+\left(\left|\alpha_{\mu}\right|+\left|\beta_{\mu}\right|\right) t^{\mu+1}+\sum_{j=\mu+1}^{k}\left(\alpha_{j} t-\alpha_{j-1}\right) t^{j}+\sum_{j=k+1}^{n}\left(\alpha_{j-1}-\alpha_{j} t\right) t^{j} \\
& +\sum_{j=\mu+1}^{l}\left(\beta_{j} t-\beta_{j-1}\right) t^{j}+\sum_{j=l+1}^{n}\left(\beta_{j-1}-\beta_{j} t\right) t^{j}+\left(\left|\alpha_{n}\right|+\left|\beta_{n}\right|\right) t^{n+1} \\
& =2\left(\left|\alpha_{0}\right|+\left|\beta_{0}\right|\right) t+\left(\left|\alpha_{\mu}\right|-\alpha_{\mu}+\left|\beta_{\mu}\right|-\beta_{\mu}\right) t^{\mu+1}+2\left(\alpha_{k} t^{k+1}+\beta_{l} t^{l+1}\right) \\
& +\left(\left|\alpha_{n}\right|-\alpha_{n}+\left|\beta_{n}\right|-\beta_{n}\right) t^{n+1} \\
& =M .
\end{aligned}
$$

The result now follows as in the proof of Theorem 1.

\section{References}

[1] K.K. Dewan, Extremal properties and coefficient estimates for polynomials with restricted zeros and on location of zeros of polynomials, Ph.D. Thesis, Indian Institute of Technology, Delhi, 1980.

[2] K.K. Dewan, M. Bidkham, On the Eneström-Kakeya theorem, J. Math. Anal. Appl. 180 (1993) 29-36.

[3] R.B. Gardner, N.K. Govil, On the location of the zeros of a polynomial, J. Approx. Theory 78 (1994) 286-292.

[4] R. Gardner, B. Shields, The number of zeros of a polynomial in a disk, J. Class. Anal. 3 (2013) 167-176.

[5] N.K. Govil, Q.I. Rahman, On the Eneström-Kakeya theorem, Tôhoku Math. Jour. 20 (1968) 126-136.

[6] I. Qasim, T. Rasool, A. Liman, Number of zeros of a polynomial (Lucanarytype) in a disk, J. Math. Appl. 41 (2018) 181-194.

[7] A. Joyal, G. Labelle, Q.I. Rahman, On the location of zeros of polynomials, Canad. Math. Bull. 10 (1967) 53-63.

[8] M. Marden, Geometry of Polynomials, Math. Surveys, No.3, Amer, Math. Soc., Providence, R.I., 1966.

[9] Q.G. Mohammad, On the zeros of the polynomials, Amer. Math. Monthly 72 (1965) 631-633.

[10] M.S. Pukhta, On the zeros of a polynomial, Appl. Math. 2 (2011) 1356-1358.

[11] Q.I. Rahman, G. Schmeisser, Analytic Theory of Polynomials, Oxford University Press, 2002. 
[12] T. Rasool, I. Ahmad, A. Liman, On zeros of polynomials with restricted coeffcients, Kyungpook Math. J. 55 (2015) 807-816.

[13] E.C. Titchmarsh, The Theory of Functions, 2nd Edition, Oxford University Press, London, 1939.

DOI: $10.7862 / \mathrm{rf} .2019 .9$

\author{
Abdullah Mir \\ email: mabdullah_mir@yahoo.co.in \\ Department of Mathematics \\ University of Kashmir \\ Srinagar, 190006 \\ INDIA
}

Abrar Ahmad

email: abrarahmad1100@gmail.com

Department of Mathematics

University of Kashmir

Srinagar, 190006

INDIA

\title{
Adil Hussain Malik
}

email: malikadil6909@gmail.com

Department of Mathematics

University of Kashmir

Srinagar, 190006

INDIA 